\title{
Impacts of Different Drying Methods on Mold Viability and Ochratoxin A Content of Grape Pomace
}

\author{
Jianmei Yu ${ }^{1,}$, , Ivy Smith ${ }^{1}$, Bernice Karlton-Senaye ${ }^{2}$, Nona Mikiashvili ${ }^{1}$, Leonard Williams ${ }^{2}$ \\ ${ }^{1}$ Department of Family and Consumer Sciences, North Carolina Agricultural and Technical State University, Greensboro, U.S.A \\ ${ }^{2}$ Center of Excellence in Post-Harvest Technologies, North Carolina Agricultural and Technical State University, Kannapolis, U.S.A
}

\author{
Email address: \\ jyu@ncat.edu(J. Yu) \\ ${ }^{*}$ Corresponding author
}

\section{To cite this article:}

Jianmei Yu, Ivy Smith, Bernice Karlton-Senaye, Nona Mikiashvili, Leonard Williams. Impacts of Different Drying Methods on Mold Viability and Ochratoxin A Content of Grape Pomace. International Journal of Applied Agricultural Sciences. Vol. 4, No. 2, 2018 , pp. $35-42$. doi: $10.11648 /$ j.ijaas.20180402.12

Received: March 9, 2018; Accepted: March 24, 2018; Published: May 2, 2018

\begin{abstract}
Value added utilization of grape pomace (GP) has been the interest of many food researchers due to its high contents in fiber and polyphenols. However, the contamination of GP by molds and ochratoxin A (OTA) present a serious safety issue to food or feed use of GP. To ensure the safety of direct usage of GP as food ingredient, this study investigated the effects of drying method on mold viability and ochratoxin A (OTA) content of GP. Pomaces of seven grape cultivars were dehydrated by freeze, room temperature and vacuum drying methods. The total population of yeast and mold colonies was enumerated using Dichloran Rose Bengal Chloramphenicol Agar (DRBC) and Dichloran Glycerol 18\% (DG18) media. The OTA was extracted by $70 \%$ methanol aqueous solution, and then quantified by an ELISA method. Regardless the grape cultivars, vacuum drying most effectively reduced the viability of mold in GP samples, while freeze-drying was the least effective method. OTA was present in all pomace samples tested but the contents of OTA in GP varied with grape variety. Vacuum drying and freeze drying significantly reduced the OTA contents of most of the pomace samples tested whereas room temperature drying increased OTA contents of all GP samples tested compared with OTA contents measured before drying. Overall, vacuum dry and freeze dry methods resulted in safer GP for food and feed use due to the greater reduction of viable molds and OTA content.
\end{abstract}

Keywords: Grape Pomace, Mold, Viability, Ochratoxin A, Drying Method

\section{Introduction}

Ochratoxin A (OTA) is a mycotoxin produced by several species of Aspergillus including A. alliaceus, A. auricomus, A. carbonarius, A. glaucus, A. melleus, and A. niger and Penicillium fungi. Our previous study confirmed the presence of OTA producing mold spores in the pomaces of some grape cultivars grown in North Carolina [1]. OTA has been shown to be nephrotoxic, hepatotoxic, teratogenic, and immunotoxic to several animal species including causing kidney and liver tumors in mice and rats [2-3], although there is no statistically significant evidence for human health risks associated with OTA exposure [4]. Ochratoxin A has been found in barley, oats, rye, wheat, coffee beans, and other plant products [5]. Due to the potential toxicity of OTA to human, both European commission and Health Canada proposed maximum permitted levels of OTA in a variety of foods and drinks [6-7]. No specific limits for OTA in foodstuffs are set in the USA.

Grape pomace (GP), the residue of grapes after wine making, contains the grape skins, pulp, and seeds. Grape pomace is a valuable source of phenolic antioxidants and dietary fiber [8], and thus may have technological applications as food ingredient for possible nutritional benefits. However, grapes are easily contaminated by molds due to its high sugar content and acidity. The presence of mycotoxin in the GP becomes a safety concern when GP is being used as a food ingredient.

According to other researchers, OTA is the main mycotoxin found in grapes, wines and grape juices [9-10]. During wine making, most OTA is removed with the pomace 
and lees [11-12]. Therefore, OTA concentration is usually low in the wine but high in the pomace. Because GP has not been widely used in foods, the safety of GP due to the presence of OTA has not been seriously evaluated. So far, only limited research has been done to assess mycotoxin level in GP [13]. Due to the potential health benefits of GP fiber and polyphenols, the interest of using GP as an ingredient in functional food development is increasing [1418]. Therefore, it is important to evaluate the presence of potential pathogenic factors such as pathogenic molds and OTA in the GP and develop method to reduce these risk factors.

OTA is a stable compound, and common food processing methods could not reduce it. It possesses a resistance to acidity and high temperatures. Thus, once foodstuffs are contaminated, it is very difficult to totally remove this molecule [19]. However, some studies shows that roasting of coffee bean resulted in 14-94\% reduction of OTA depending on the degree of roasting and dark roasting resulted in higher OTA reduction [20-21]. In addition, due to the fact that OTA producing molds grow at high humidity, the rapid removal of moisture in grape pomace should significantly reduce the viability of mold, thus the content of OTA. Due to the increasing interest in utilizing GP as food ingredient, it is important to investigate the effects of processing methods on the OTA level in the pomace to explore the methods that can reduce the viability of mold spores and the OTA content in grape pomace. The objective of this study was to investigate drying methods on the viability of mold and OTA level of pomace of different grape cultivars and to compare the OTA content of seeds and skins of grape pomace.

\section{Materials and Methods}

\subsection{Materials}

Wet pomaces of seven grape cultivars including Muscadine Noble, Muscadine Carlos, Cabernet Franc, Cabernet Sauvignon, Merlot, Sangiovese and Chardonnay were collected from two North Carolina wineries (USA) in late September and early October of 2014. All pomace samples were collected within one hour of press. Among these pomace samples, Muscadine Noble, Cabernet Franc, Cabernet Sauvignon, Merlot and Sangiovese were red, Muscadine Carlos was green-brown and Chardonnay was green. The collected pomace of each grape variety was split into several bags $\left(6 \times 10\right.$ inch) which were stored at $-20{ }^{\circ} \mathrm{C}$ until used, to prevent repeated freezing and thawing. Dichloran Rose Bengal Chloramphenicol Agar (DRBC), Dichloran Glycerol 18\% (DG18) and Potato Dextrose Agar (PDA) plates were purchased from Thermo Scientific (Suwanee, GA USA) and used for the identification of mold and yeast. AgraQuant Ochratoxin A kit was purchased from Romer Labs, Inc (Union, MO, USA). The kit included antibody coated well strips, dilution well strips, conjugated solution, substrate solution, stop solution and a set of OTA standard solutions.

\subsection{Drying of Grape Pomace}

About 500g of each GP sample was freeze dried (FD), room temperature dried (RTD) and vacuum dried (VD). For freeze drying, GP samples were spread in plastic trays to about one inch thick, frozen at $-80^{\circ} \mathrm{C}$ overnight, then dried in a Labconco Freeze Dryer for $48 \mathrm{hrs}$. For vacuum drying, samples were spread into one inch layer in coated metal trays and dried in pre-heated a Isotemp vacuum oven (Fisher Scientific, USA) for 24 hours at $80^{\circ} \mathrm{C}$. For room temperature drying, samples were spread into half inch layer in plastic trays, placed in a well ventilated room with temperature maintained at about $22^{\circ} \mathrm{C}$ for one week. Dried samples were ground into powders, bagged in moisture proof plastic bags and stored at $4{ }^{\circ} \mathrm{C}$.

The moisture contents of all pomace samples were measured by vacuum oven. Briefly, $5.00 \mathrm{~g}$ of wet pomace was weighed in a known weight aluminum weight boat and dried in an Isotemp vacuum oven (Fisher Scientific, USA) for 24 hrs at $80^{\circ} \mathrm{C}$. The moisture content was calculated as weight loss. The measurement was triplicate for the pomace of each grape variety. The moisture contents of GP samples were used to convert the OTA concentration from wet base (WB) to dry base (DB).

$$
\mathrm{OTA} \mu \mathrm{g} / \mathrm{kg} \mathrm{DB}=\mathrm{OTA} \mu \mathrm{g} / \mathrm{kg} \mathrm{WB} /(1 \text {-moisture })
$$

\subsection{Determination of Total Fungal Counts in GP}

Five grams $(5.00 \mathrm{~g})$ of each dry GP were mixed with $25 \mathrm{ml}$ of $0.1 \%$ peptone water and blended with a stomach blender for 2 minutes, serially diluted and $100 \mu \mathrm{l}$ of appropriate diluents were spread plated in duplicates on DRBC and DG18 plates. The total population of yeast and mold colonies was enumerated and results were reported in colony forming units CFU/ml from average fungal counts. To ensure the determination of accurate fungal population 3.00 of each dry GP samples was also directly plated by aseptically weighing the samples and directly adding each sample to both DRBC and DG18 agar media. Both sets of samples were then incubated at $25^{\circ} \mathrm{C}$ for 7 days. The total population of yeast and mold colonies was enumerated and results were reported in colony forming units $\mathrm{CFU} / \mathrm{g}$ GP from average fungal counts.

\subsection{Determination of $O T A$ in $G P$}

For dry GP samples, $5.00 \mathrm{~g}$ of dry powder was mixed with $25 \mathrm{ml}$ of $70 \%$ methanol aqueous solution and stirred at room temperature for 60 minutes. For wet GP samples, $50.00 \mathrm{~g}$ of each variety was mixed with $50 \mathrm{ml}$ of DI water and homogenized into slurry using a Polytron Homogenizer PTMR2100 (Kinematic AG, Switzerland). Five grams of homogenized slurry was extracted with $10 \mathrm{ml}$ of non-diluted methanol. Samples were centrifuged, and then filtered through $0.45 \mathrm{um} \mathrm{GF/CA} \mathrm{filter.} \mathrm{The} \mathrm{volumes} \mathrm{of} \mathrm{filtrates} \mathrm{were}$ recorded. The filtrates were used for OTA analysis. The extraction of each GP variety was conducted in triplicate.

OTA concentration in each extract was quantified using 
AgraQuant Ochratoxin A kit (Romer Labs, Inc). The AgraQuant ${ }^{\circledR}$ Ochratoxin Assay has been validated by the vendor for barley, beer, cocoa, corn, cereal, green coffee, milo, soybeans, wheat and wine. Briefly, $100 \mu \mathrm{l}$ of each extract was diluted with $200 \mu \mathrm{l}$ of conjugated solution in the mixing wells by pipetting up and down several times. The diluted samples $(100 \mu \mathrm{l})$ were added to antibody coated wells and incubated for $10 \mathrm{~min}$ at room temperature. The wells were washed 5 times with deionized water, tap dried, then $100 \mu 1$ of substrate was added into the well. After $5 \mathrm{~min}$ incubation, $100 \mu$ l of stop solution was added to each well to stop activity. The ELISA strips were read using a HTX Synergy Microplate Reader (BioTek, Winooski, VT, USA) at $450 \mathrm{~nm}$ using $630 \mathrm{~nm}$ as differential filter. A standard curve was developed using a set of OTA standard solutions by the same procedure. The OTA concentrations of extracts were expressed as $\mathrm{ng} / \mathrm{ml}$ extract, and the OTA contents of GP samples were calculated according to sample weight, volumes of extracts and moisture content, and expressed as $\mu \mathrm{g} / \mathrm{kg}$ dry mass (pomace).

\subsection{Determination of OTA in Grape Seeds and Grape Skins}

To evaluate whether grape skins or seeds were more contaminated with OTA, the GP samples dried by different methods were separated into seeds and skins manually. The OTA contents of seeds and skins were extracted and determined as described in section 2.4.

\subsection{Data Analysis}

OTA data collected were expressed as mean \pm standard deviation. The effects of drying methods on OTA content in GP were compared by Duncan multiple comparison using SAS version 9.2 (SAS Institute Inc., Cary, NC, USA).

\section{Results and Discussion}

\subsection{Effects of Drying Methods on Viability of Yeast and Mold in Grape Pomace}

Our previous study showed the presence of ochratoxigenic molds in the pomace of certain grape cultivars [1]. Among all molds identified in Chardonnay pomace, A. niger, $A$. carbonarius and A. fumigatus contributed to $81.1 \%, 13.51$ and $5.39 \%$ of the contamination. Overall, the pomace produced from Chardonnay and Merlot grape cultivars were mostly populated with all three Aspergillus strains detected. No mold was detected in pomaces derived from Cabernet Franc and Sangiovese grape cultivars. In addition, yeast was also found in wet GP samples.

Table 1 shows the effects of different drying methods on the viability of mold and yeast in GP samples detected by DG 18 medium. For room temperature dried GP samples, no yeast and mold were found in Muscadine Noble and Carlos pomaces, large number of yeast colonies and small number of mold colonies (1-2 $\mathrm{CFU} / \mathrm{ml}$ ) were found in Cabernet Sauvignon, Cabernet Franc and Merlot pomaces, but large number of mold colonies were found in Chardonnay pomace $(>300$ $\mathrm{CFU} / \mathrm{ml}$ ). For vacuum dried pomace samples, low numbers of mold colonies were found in Cabernet Franc and Chardonnay pomaces but yeast were found in all pomace samples at a much larger number. Larger numbers of mold colonies $(2-300 \mathrm{CFU} / \mathrm{ml})$ were found in freeze dried pomace samples except Muscadine Noble. The counts of yeast colonies in freeze dried Cabernet Franc, Sangiovese, Merlot and Chardonnay pomaces were higher. The highest mold counts of dry Chardonnay pomace corresponded to high mold load of wet sample found in our previous study [1].

Table 1. Fungal populations of grape pomace samples (CFU/ml) determined by indirect plating method using DG18 medium plates (incubated at $25{ }^{\circ} \mathrm{C}$ for 7 days on DG18).

\begin{tabular}{|c|c|c|c|c|c|c|}
\hline \multirow{2}{*}{ Grape Pomace } & \multicolumn{2}{|c|}{ Room Temperature Dried } & \multicolumn{2}{|c|}{ Vacuum Dried } & \multicolumn{2}{|c|}{ Freeze Dried } \\
\hline & Yeast & Mold & Yeast & Mold & Yeast & Mold \\
\hline Noble & ND & ND & 10 & ND & 2 & ND \\
\hline Carlos & ND & ND & 34 & ND & ND & 4 \\
\hline Sauvignon & 86 & 2 & 212 & ND & ND & 3 \\
\hline Franc & $>300$ & ND & 94 & 3 & 37 & 2 \\
\hline Sangiovese & 7 & 1 & 20 & ND & 55 & 4 \\
\hline Chardonnay & ND & $>300$ & 30 & ND & $>300$ & $>300$ \\
\hline
\end{tabular}

ND-not determined; NG-no growth, $<10=$ less than 10 colonies $/ \mathrm{ml}$.

Table 2 shows yeast and mold populations of GP samples dried by different methods on DRBC medium. The yeasts existed in all GP samples tested regardless the drying method, but the mold counts were much lower and varied with drying method greatly. For room temperature dried GP samples, mold was only detected in Cabernet Sauvignon and
Chardonnay pomaces at levels of 2 and $12 \mathrm{CFU} / \mathrm{ml}$, respectively. For vacuum dried samples, only Merlot pomace showed $3 \mathrm{CFU} / \mathrm{ml}$. The mold was detected in most of the freeze dried GP samples at 1-5 CFU/ml. The highest mold population was observed in room temperature dried Chardonnay pomace. 
Table 2. Fungal populations of grape pomace samples (CFU/ml) determined by indirect plating method on DRBC medium (incubated at $25{ }^{\circ} \mathrm{C}$ for 7 days).

\begin{tabular}{|c|c|c|c|c|c|c|}
\hline \multirow{2}{*}{ Grape pomace } & \multicolumn{2}{|c|}{ Room Temperature Dried } & \multicolumn{2}{|c|}{ Vacuum Dried } & \multicolumn{2}{|c|}{ Freeze Dried } \\
\hline & Yeast & Mold & Yeast & Mold & Yeast & Mold \\
\hline Noble & 3 & ND & 5 & ND & 53 & 1 \\
\hline Carlos & 50 & ND & 35 & ND & ND & 3 \\
\hline Franc & $>300$ & ND & 64 & ND & 12 & 1 \\
\hline Sangiovese & 6 & ND & 10 & ND & 40 & 1 \\
\hline Merlot & 175 & ND & 53 & 3 & 9 & 5 \\
\hline
\end{tabular}

ND-not determined.

The yeast count in the GP seems varied with both grape cultivar and drying method. Yeasts represent the most important group of microorganisms to wine makers. The yeasts, naturally present on the grape surface, transform sugars (glucose and fructose) in musts into ethanol and carbon dioxide. Generally, the major species identified on the surface of grapes are non-Saccharomyces yeasts which impact the aroma and flavor composition of the finished wine [22]. Some wineries add yeast ( $S$. cerevisiae) to the musts to the total population of $5 \times 10^{6}$ cells $/ \mathrm{ml}$ to prevent undesirable compounds formation [23]. At the later stage of fermentation, the viable yeast population decreases because of the lack of nutrients and the increased level of ethanol, but some yeast remain active. Therefore, it is not surprising to see large number of yeast colonies in the wet and dry pomace. Because yeasts do not produce toxins, their presence does not generate a safety issue to GP.

Among all vacuum dried GP samples, only Cabernet Franc and Merlot showed mold growth, but all freeze dried GP samples showed mold growth on both DG18 and DRBR plates. Regardless the grape cultivars, vacuum drying most effectively reduced the viability of mold in GP samples, while freeze drying was the least effective method. Among all pomace samples, room temperature dried and freeze dried Chardonnay pomace showed highest fungal population which might be related to its high fungi load as evidenced in our previous study [1].

To further confirm the presence and absence of molds in the dried GP samples, direct plating method was conducted. Dried pomace powders were directly spread on the surface of DRBC medium plates. The results did not show difference in mold counts among pomace samples dried by different methods (Table 3). Molds were not detected in all Noble, Carlos, Cabernet Sauvignon and Sangiovese pomaces. However, 6 $\mathrm{CFU} / \mathrm{g}$ of molds were detected in room temperature dried Cabernet Franc pomace and vacuum dried Merlot pomace, 1 $\mathrm{CFU} / \mathrm{g}$ was observed for Chardonnay pomace. High yeast count was observed in room temperature dried Sangiovese pomace. Low yeast population of other GP samples plated by direct plating might be caused by the low moisture.

Table 3. Fungal populations of grape pomace samples (CFU/g) determined by spread (direct) plating of dry GP powders on DRBC (incubated at $25{ }^{\circ} \mathrm{C}$ for 7 days).

\begin{tabular}{|c|c|c|c|c|c|c|}
\hline \multirow{2}{*}{ Grape Pomace } & \multicolumn{2}{|c|}{ Room Temperature Dried } & \multicolumn{2}{|c|}{ Vacuum Dried } & \multicolumn{2}{|c|}{ Freeze Dried } \\
\hline & Yeast & Mold & Yeast & Mold & Yeast & Mold \\
\hline Noble & - & - & - & - & - & - \\
\hline Carlos & - & - & - & - & - & - \\
\hline Sauvignon & - & - & - & - & - & - \\
\hline Franc & 8 & 6 & - & - & 7 & 2 \\
\hline Sangiovese & $>300$ & - & 6 & - & - & - \\
\hline Chardonnay & - & - & - & - & - & 1 \\
\hline
\end{tabular}

The OTA producing molds grow in the temperature range $10-45^{\circ} \mathrm{C}$ and water activity range of $0.80-0.99$ [24]. Depending on the species, the optimal growth temperature for ochratoxigenic mold could be $15-25^{\circ} \mathrm{C}$ [25]. The highest growth rate of $A$. carbonarius was reported to be $25-35^{\circ} \mathrm{C}$ $[24,26]$. Another study shows the optimal growth temperature and water activity of $A$. carbonarius and $A$. niger are $23-33^{\circ} \mathrm{C}$ and $0.95-0.99$ respectively [27]. Both mold growth and OTA accumulation are favored by high moisture $(24,27)$. At freeze drying temperature, molds could not grow and could die when the moisture was below the minimum requirement for growth and survival. At vacuum drying temperature used in this study, molds could not survive once the center of GP reaches $80^{\circ} \mathrm{C}$. The fast drying method such as vacuum drying should quickly reduce the moisture content which is very important for the survival of mold cells, thus decreasing the possibility for mold to continually produce OTA during storage of pomace. The room temperature was about $22^{\circ} \mathrm{C}$ which fell into the optimal growth temperature of ochratoxigenic molds. Because it took one week to dry GP at room temperature, the molds in the pomace could grow and produce OTA although this could not be seen with eyes. 


\subsection{OTA Content of Fresh Grape Pomace}

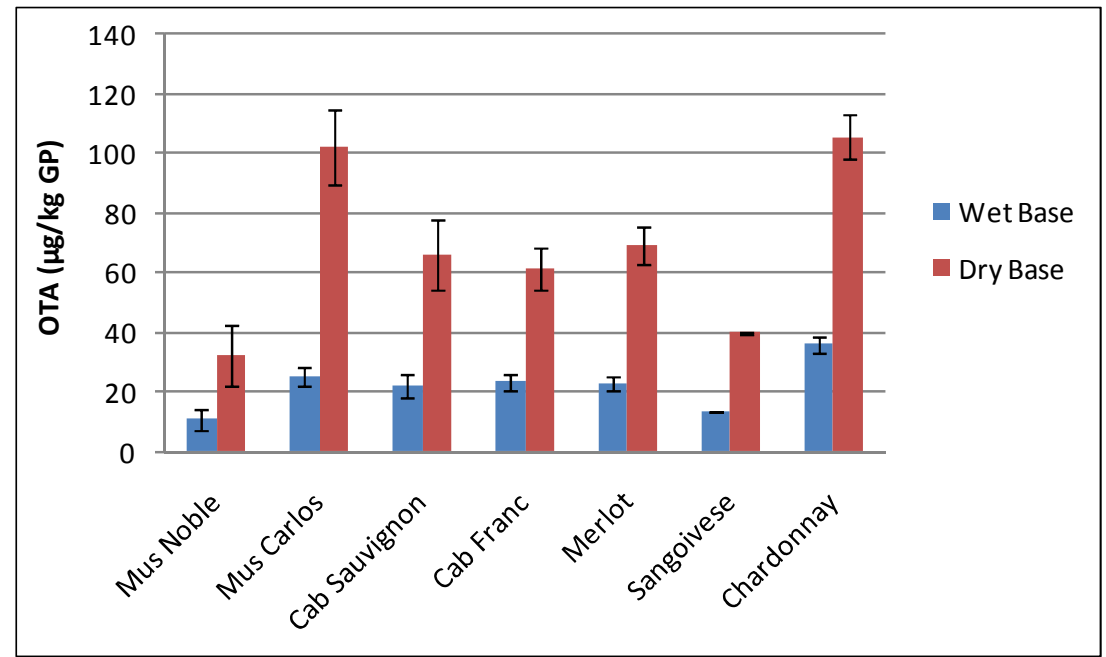

Figure 1. OTA contents of grape pomace samples tested (OTA was determined using wet pomace samples).

Figure 1 shows that OTA were present in all pomace samples tested but the contents of OTA in GP varied with grape variety. The moisture contents of wet GP samples of Muscadine Noble, Muscadine Carlos, Cabernet Franc, Cabernet Sauvignon, Merlot, Sangiovese and Chardonnay were $66.20,75.06,66.06,61.89,66.80,66.12$, and $65.95 \%$, respectively. The OTA contents of wet GP samples was in the range of $11-35 \mu \mathrm{g} / \mathrm{kg}$ on the wet basis (WB), but $32-105$ $\mu \mathrm{g} / \mathrm{kg}$ on the dry basis (DB). Muscadine Noble and Sangiovese pomaces had lowest OTA contents (11.0 and 13.5 $\mu \mathrm{g} / \mathrm{kg} \mathrm{WB}$ or 32.5 and $35.8 \mu \mathrm{g} / \mathrm{kg} \mathrm{DB}$, respectively), while Muscadine Carlos and Chardonnay pomaces had highest OTA contents $(25.53$ and $35.96 \mu \mathrm{g} / \mathrm{kg} \mathrm{WB}$ or 102.5 and $105.6 \mu \mathrm{g} / \mathrm{kg} \mathrm{DB}$, respectively). The OTA contents of the rest of tested GP samples were about $22 \mu \mathrm{g} / \mathrm{kg}$ WB or $60 \mu \mathrm{g} / \mathrm{kg}$ WB. The high OTA content of Chardonnay pomace is corresponding to its high mold population. Because the OTA limit in GP was not established, the maximal GPA allowance of dry fruit $(10 \mu \mathrm{g} / \mathrm{kg})$ set by European Commission was used as the upper limit of dry GP [5]. The results show that all dry GP samples had OTA contents higher than the maximal allowance of $10 \mu \mathrm{g} / \mathrm{kg}$, and the dry GP cannot be consumed alone.

\subsection{Effects of Drying Methods on OTA Contents of Grape Pomace}

Drying method has significant effects on the OTA content of GP (Figure 2). Compared with OTA detected in the wet pomace, vacuum drying at $80^{\circ} \mathrm{C}$ for 24 hours and freeze drying for 48 hours significantly reduced the OTA contents of Cabernet Franc, Cabernet Sauvignon, Merlot, Sangiovese and Chardonnay pomaces, but increased the OTA contents of Muscadine Noble and Carlos pomaces. Room temperature drying only decreased OTA contents in GP samples of Sauvignon, Merlot and Chardonnay compared with OTA contents measured before drying. The OTA contents were in the range of $20-30 \mathrm{um} / \mathrm{kg}$ for vacuum and freeze dried Cabernet Sauvignon, Merlot and Sangiovese pomace samples, and $40-60 \mu \mathrm{g} / \mathrm{kg}$ in vacuum and freeze dried Muscadine Noble, Cabernet Franc and Chardonnay pomace sample. Muscadine Carlos pomace showed highest OTA contents (130-168 $\mu \mathrm{g} / \mathrm{kg}$ GP) regardless of drying method. During room temperature drying, the growth of mold was visualized in Muscadine Carlos pomace but not the other pomace. This could be why the OTA content of Muscadine Carlos pomace was higher than other pomace samples although the molded pomace was discarded before grinding.

Vacuum drying quickly heated the GP to $80^{\circ} \mathrm{C}$ and rapidly reduced the moisture of GP which should kill OTA producing molds in a short period of time as shown by the extremely low mold counts of vacuum dried GP samples (Table 2 and 3). Therefore, the possibility of producing OTA during vacuum drying is low. However, the OTA contents of vacuum dried Muscadine Noble and Carlos GP samples were higher than that of control. This is most likely due to the thick skin of Muscadine grape pomace. Freeze drying was conducted at $-5^{\circ} \mathrm{C}$. Although it took 48 hours to reach the desired dryness, molds should be in the inactive state and should not produce OTA during this period because the temperature for OTA production is $15-45^{\circ} \mathrm{C}$ with $35-37{ }^{\circ} \mathrm{C}$ being the optimum temperature for the growth and $20{ }^{\circ} \mathrm{C}$ being the optimal temperature of OTA production [24-25]. According to previous studies, the major molds responsible for the OTA production in GP are A. niger and $A$. carbonarius $[1 ; 26]$. Our room temperature drying was conducted at $22^{\circ} \mathrm{C}$ which is near the optimal OTA production temperature of $A$. carbonarius and A. Niger $\left(15-20^{\circ} \mathrm{C}\right)$ [24, $28-30 ; 27]$. Therefore, it is easy to understand the increase of OTA in some room temperature dried GP samples, particularly, the thick skin pomace. 


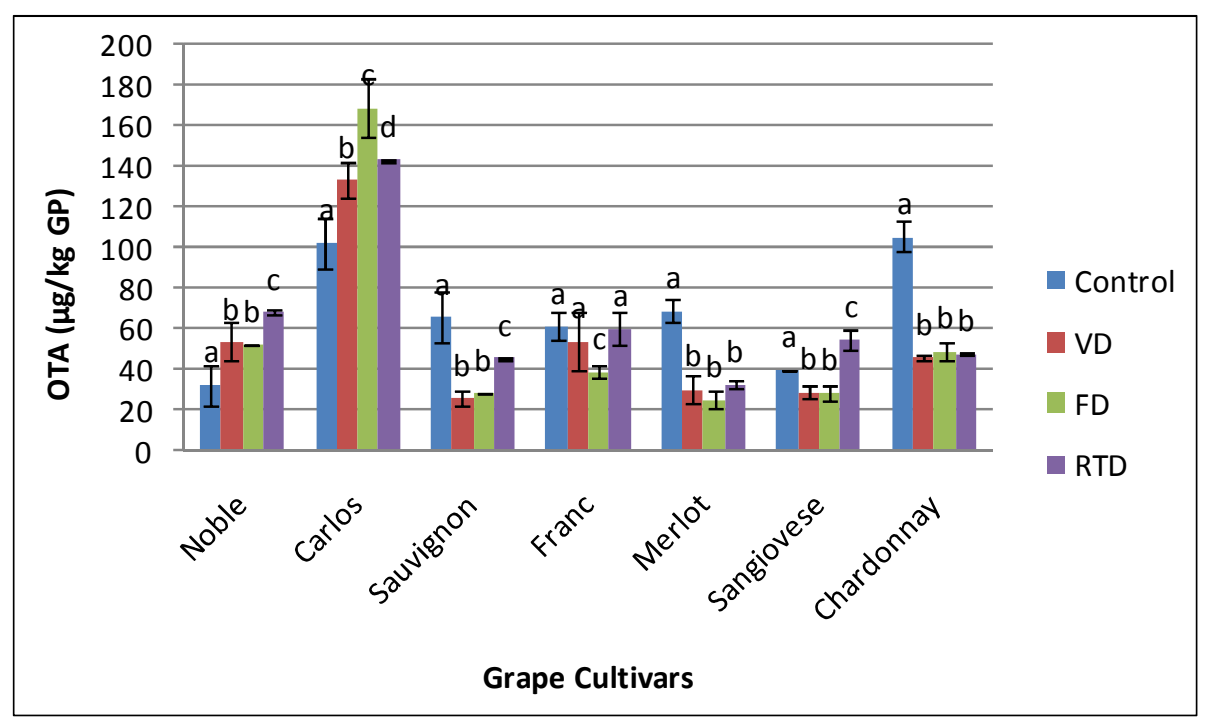

Figure 2. Effect of drying methods on OTA content of grape pomace (Control-OTA measured using wet pomace and converted into OTA $\mu \mathrm{g} / \mathrm{kg}$ dry pomace), Control-wet GP (OTA content was calculated based on dry mass), VD-vacuum drying, RT-room temperature drying, FD-Freeze drying (For same grape cultivar, bars with same label are not significantly different at $P<0.05)$.

OTA is a highly stable mycotoxin which possesses a resistance to acidity and high temperatures [19]. Most of the food processes have variable effects on mycotoxins, with those that utilize the highest temperatures having greatest effects [31]. It was reported that roasting could reduce the content of OTA in coffee beans by up to $97 \%$ depending on the temperature and particle size and extrusion was less efficient [32]. The drying methods used in this study are very mild comparing to roasting, thus the effects of drying on OTA reduction is limited. However, quick drying after fermentation will preserve GP from further contamination due to mold growth and OTA production.

\subsection{OTA Contents in Grape Skins and Seeds}

Figure 3 shows that the impact of drying methods on the OTA contents of skins and seeds of GP samples is inclusive. The OTA contents of vacuum, freeze and room temperature dried GP skins were 27-301, 47-302 and 24-256 $\mu \mathrm{g} / \mathrm{kg}$, respectively, whereas the OTA contents of vacuum, freeze and room temperature dried GP seeds were 32 -229, 64-275 and 21-303 $\mu \mathrm{g} / \mathrm{kg}$, respectively. Vacuum dried (VD) skins of Muscadine Noble, Muscadine Carlos and Cabernet Sauvignon pomaces had lower OTA contents than the skins dried by other methods ( $\mathrm{P}<0.05)$, while room temperature dried (RT) skins of other GP samples had lower OTA than the vacuum and freeze dried skins $(\mathrm{P}<0.05)$ (Figure $3 \mathrm{~A})$. For the seeds, 3 out of 7 freeze dried (FD) samples and 3 out 7 room temperature dried samples showed highest OTA (Figure 3B). Regardless of drying methods, the skins of Muscadine Carlos pomace had highest OTA contents (194-256 $\mu \mathrm{g} / \mathrm{kg}$ ) among all skin samples (Figure 3A), while seeds of Chardonnay pomace showed highest OTA content $(191-303 \mu \mathrm{g} / \mathrm{kg})$ among all seed samples (Figure 3B). Overall, vacuum dried skins and seeds showed lower OTA content. However, vacuum drying may result in significant loss of anthocyanins due to the heat sensitive nature of most of these polyphenols [33].

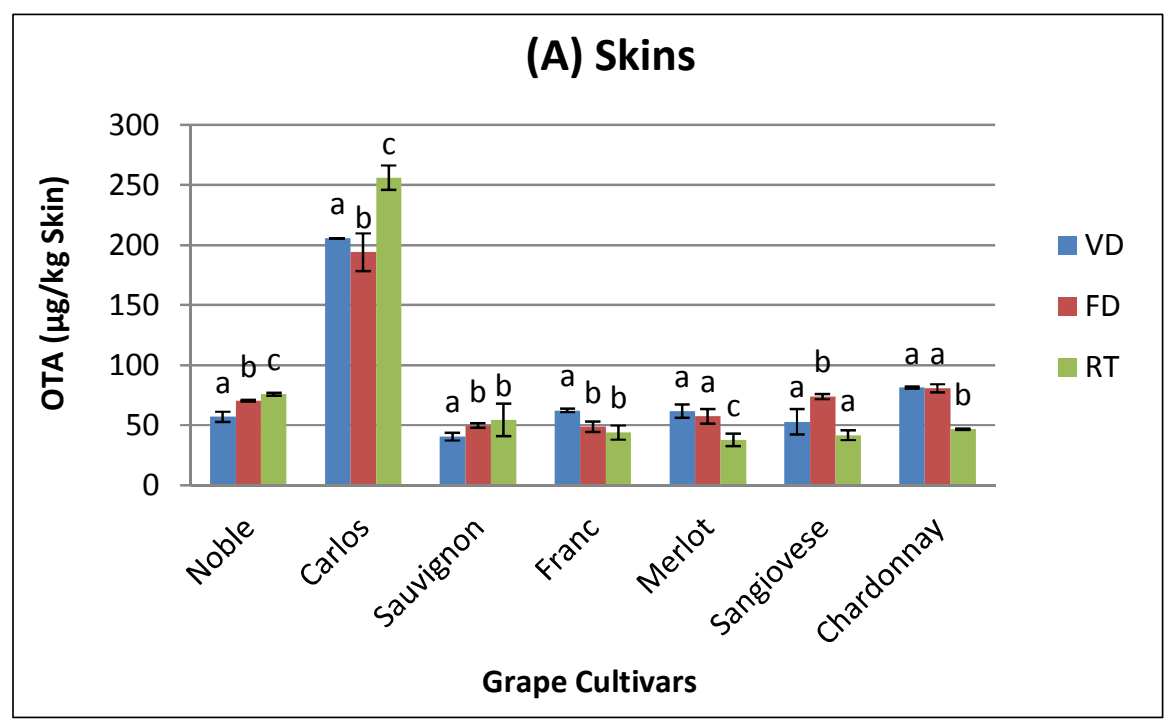




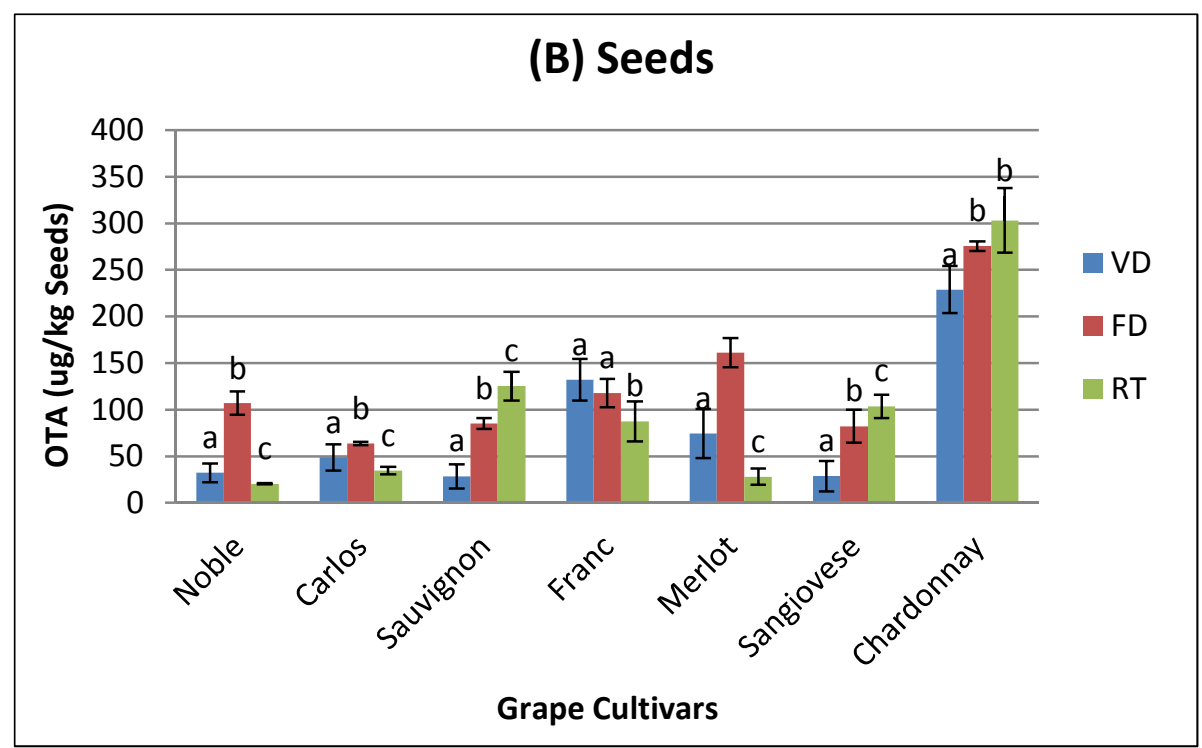

Figure 3. OTA contents in the skins and seeds of grape pomaces as affected by drying methods (VD-vacuum drying, RT-room temperature drying, FD-Freeze drying). (For same grape cultivar, bars with same label are not significantly different at $P<0.05$ ).

\section{Conclusion}

The degree of mold contamination and OTA content of GP varied with the grape cultivars and agricultural practice. Drying methods of GP had great impact on the microbial load and ochratoxin A content. Fast drying after press of GP greatly reduced viable mold count and OTA content in the pomace. For most of grape varieties tested, vacuum drying and freeze drying resulted in comparable OTA content in dried GP, but vacuum dried samples showed lower OTA in both skins and seeds. Vacuum drying could destroy mold spores but might damage polyphenols. In contrast, freeze drying could not destroy mold spores although it preserved polyphenol antioxidant in the pomace. Vacuum drying is faster and cheaper than freeze drying. Therefore, from safety of GP and economy point of view, vacuum drying method is recommended. The limitation of this study is that the OTA content of GPs was determined by one ELISA method and the reproducibility of the ELISA method was not very good. It is important to select or develop a more accurate and reproducible method for OTA analysis. In addition, it is necessary to develop a more effective method to reduce OTA content of GP to improve the safety of utilizing GP as a food ingredient.

\section{Author Disclosure Statement}

There is no conflict interest.

\section{Acknowledgements}

This study was financially supported by a Capacity Building grant (Award number: 2013-38821-21147) sponsored by the National Institute of Food and Agriculture, the US Department of Agriculture.

\section{References}

[1] Karlton-Senaye, B. D., Yu, J., \& Williams, L. L. (2015). Morphological and molecular characterization of ochratoxin a producing black Aspergilli from grape pomace. Journal of Food Research, 4, 39-50.

[2] Varga, J., Kocsubé, S., Péteri, Z., Vágvölgyi, C., \& Tóth, B. (2010). Chemical, physical and biological approaches to prevent ochratoxin induced toxicoses in humans and animals. Toxins, 2, 1718-1750.

[3] Tamás Kőszegi T., \& Poór, M. (2016). Ochratoxin A: Molecular interactions, mechanisms of toxicity and prevention at the molecular level. Toxins, 8, 111-135.

[4] Bui-Klimke, T. R., \& Wu, F. (2015). Ochratoxin A and human health risk: a review of the evidence. Critical Reviews in Food Science and Nutrition, 55, 1860-1869.

[5] Kolakowski, B., O'rourke, S. M., Bietlot, H. P., Kurz, K., \& Aweryn, B. (2016). Ochratoxin A Concentrations in a variety of grain-based and non-grain-based foods on the Canadian retail market from 2009 to 2014. Journal of Food Protection, $79,2143-2159$.

[6] EC 1881, 2006. COMMISSION REGULATION (EC) No 1881/2006 of 19 December 2006 setting maximum levels for certain contaminants in foodstuffs. 2006R1881-EN01.07.2014-013.001-2.

[7] Health Canada. (2009). Information document on health Canada's proposed maximum limits (standards) for the presence of the mycotoxin Ochratoxin A in foods. Safety, B. o. C. Ed., Ottawa. www.hc-sc.gc.ca/fn-an/consult/ limits-maxseuils/myco_consult_ochra-eng.php.

[8] Yu, J., \& Ahmedna, M. (2013). Functional components of grape pomace: Their compositions, biological properties and potential applications. International Journal of Food Science and Technology, 18, 221-237. 
[9] Hocking, A. D., Leong, S. L., Kazi, B. A., Emmett, R. W., \& Scott, E. S. (2007). Fungi and mycotoxins in vineyards and grape products. International Journal of Food Microbiology, $119,84-88$

[10] Terra, M. F., Prado, G., Pereira, G. E., Ematné H. J., \& Batista L. R. (2013). Detection of ochratoxin A in tropical wine and grape juice from Brazil. Journal of Science of Food Agriculture, 93, 890-894.

[11] Ratola, N., Martins, L., \& Alves, A. (2004). Ochratoxin A in wine-assessing global uncertainty associated with the results. Analytica Chimica Acta, 513, 319-324.

[12] Fernandes, A., Ratola, N., Cerdeira, A., Alves, A., \& Venâncio, A. (2007). Changes in ochratoxin A concentration during winemaking. American Journal of Enology and Viticulture, 58, 92-96.

[13] Solfrizzo, M., Panzarini, G., \&Viscontij, A. (2008). Determination of ochratoxin A in grapes, dried vine fruits, and winery byproducts by high-performance liquid chromatography with fluorometric detection (HPLC-FLD) and immunoaffinity cleanup. Journal of Agricultural and Food Chemistry, 56, 11081-11086.

[14] Sánchez-Alonso, I., Jiménez-Escrig, A., Saura-Calixto, F., \&Borderías, A. J. (2007). Effect of grape antioxidant dietary fiber on the prevention of lipid oxidation in minced fish: Evaluation by different methodologies. Food Chemistry, 101, 372-378.

[15] Altan, A., McCarthy, K. L., \& Maskan, M. (2008). Twinscrew extrusion of barley-grape pomace blends: extrudate characteristics and determination of optimum processing conditions. Journal of Food Engineering, 89, 24-32.

[16] Mildner-Szkudlarz, S., Zawirska-Wojtasiak, R., Szwengiel A., \& Pacyński, M. (2011). Use of grape by-product as a source of dietary fibre and phenolic compounds in sourdough mixed rye bread. International Journal of Food Science and Technology, $46,1485-1493$.

[17] Özvural, E. B. \&Vural, H. (2011). Grape seed flour is a viable ingredient to improve the nutritional profile and reduce lipid oxidation of frankfurters. Meat Science, 88, 179-183.

[18] Smith, I. and Yu, J. (2015). Nutritional and sensory quality of bread containing different cultivars and quantities of grape pomace. EC Nutrition, 2, 291-301.

[19] Khoury, A., \& Atoui, A. (2010). Ochratoxin A: General overview and actual molecular status. Toxins, 2, 461-493.

[20] Romani, A., Pinnavaia, G. G., and Rosa, M. D. (2003). Influence of roasting levels on ochratoxin A content in coffee. Journal of Agricultural and Food Chemistry, 51, 5168-5171.

[21] Pérez de Obanos, A., González-Peñas, E., \& López de Cerain, A. (2005). Influence of roasting and brew preparation on the ochratoxin A content in coffee infusion. Food Additives and Contaminants. 22, 463-471.

[22] UC Davis (2014). Diversity of wine yeast. (http://viticulture.ucdavis.edu/industry/enology/winemicro/wi neyeast/diversity. html). Retrieved on July 21, 2017.

[23] Zamora, F. (2009). Biochemistry of alcoholic fermentation. In: Wine Chemistry and Biochemistry (M. Victoria MorenoArribas M. Carmen Polo Editors). Springer Science+Business Media, LLC, New York, USA.

[24] Bellí, N., Ramos, A. J., Coronas, I., Sanchis, V., \& Marín, S. (2005). Aspergillus carbonarius growth and ochratoxin A production on a synthetic grape medium in relation to environmental factors. Journal of Applied Microbiology, 98, 839-44.

[25] Alborch, L. Bragulat, M. R., Abarca M. L., \& Cabañes F. J. (2011). Temperature and incubation time effects on growth and ochratoxin A production by Aspergillus sclerotioniger and Aspergillus lacticoffeatus on culture media. Letters in Applied Microbiology, 52, 208-212.

[26] Battilani, P., and Camardo, L. M. (2015). OTA-grapes: a mechanistic model to predict ochratoxin a risk in grapes, a step beyond the systems approach. Toxins 2015, 7, 3012-3029.

[27] Passamani, F. R., Hernandes, T., Lopes, N. A., Bastos, S. C., Santiago, Cardoso, M. D., \& Batisa L. R. (2014). Effect of temperature, water activity, and $\mathrm{pH}$ on growth and production of ochratoxin A by Aspergillus niger and Aspergillus carbonarius from Brazilian grapes. Journal of Food Protection, 77, 1947-1952. doi: 10.4315/0362-028X. JFP-13495.

[28] Mitchell, D., Parra, R., Aldred, D., \& Magan, N. (2004). Water and temperature relations of growth and ochratoxin A production by Aspergillus carbonarius strains from grapes in Europe and Israel. Journal of Allied Microbiology, 97, 439445.

[29] Tassou, C. C., Natskoulis, P. I., Panagou, E. Z., Spiropoulos, A. E., \& Magan, N. (2007). Impact of water activity and temperature on growth and ochratoxin A production of two Aspergillus carbonarius isolates from wine grapes in Greece. Journal of Food Protection, 70, 2884-2888.

[30] Tassou, C. C., Natskoulis, P. I., Magan, N., and Panagou, E. Z. (2009). Effect of temperature and water activity on growth and ochratoxin A production boundaries of two Aspergillus carbonarius isolates on a simulated grape juice medium. Journal of Applied Microbiology, 107, 257-268.

[31] Bullerman, L. B., \& Bianchini, A. (2007). Stability of mycotoxins during food processing. International Journal of Food Microbiology, 119, 140-146.

[32] Karlovsky, P., Suman, M., Berthiller, F., DeMeester, J., Eisenbrand, G., Perrin, I., Isabelle, P., Oswald, I. P., Speijers, G., Chiodini, A., Recker, T., Dussort, P. (2016). Impact of food processing and detoxification treatmentson mycotoxin contamination. Mycotoxin Research, 32, 179-205.

[33] Yu, J. (2014). Thermal stability of major classes of polyphenols in skins, seeds and stems of grape pomace. In "Grapes: Production, Phenolic Composition and Potential Biomedical Effects", Jose de Sousa Camara (ed), pp: 273-285. Nova Science Publishers Inc. Hauppauge, NY. 